Bull. Egypt. Soc. Physiol. Sci. 40(1), 69- 83

\author{
Bull. of Egyp. Soc. Physiol. Sci. \\ (Official Journal of Egyptian Society for Physiological Sciences) \\ (pISSN: 1110-0842; eISSN: 2356-9514)
}

\title{
The Effect of Subdiaphragmatic Vagotomy on Lipid Profile and Insulin Resistance in Diabetic Obese Rats
}

\author{
Authors: Dina Sebaei ${ }^{1}$, Shimaa Mohammed Yousof ${ }^{1}$, Mohamed Abdo ${ }^{1}$ \\ ${ }^{1}$ Department of Medical Physiology, Faculty of Medicine, Suez Canal University, Ismailia
}

Received: 1 July 2019

Accepted: 23 August 2018

Available online: 1 Jan 2020

Keywords

- Vagotomy

- Insulin resistance

- Hyperlipidemia

- Glucose tolerance test

\section{Abstract}

The aim of the study was to assess of the effect of subdiaphragmatic vagotomy on lipid profile and insulin resistance in diabetic obese rat model. Fifty Male Albino rats 200 $\pm 50 \mathrm{~g}$ were divided into 5 groups 10 per each. Subdiaphragmatic vagotomy and sham operations were applied on diabetic obese rat model induced by high fat diet for 5 weeks and low dose of streptozotocin $(30 \mathrm{mg} / \mathrm{kg})$. After 5 weeks, serum samples were collected for confirmation of hyperlipidemia and lee obesity index was measured. At the end of the experiment, oral glucose tolerance test, lee obesity index, lipid profile, fasting insulinemia, fasting glucose, insulin resistance (HOMA-IR) and histopathology of pancreases were assessed. Results revealed that Subdiaphragmatic vagotomy significantly improved hyperlipidemia as it normalized plasma triglyceride level, decreased plasma cholesterol level and increased plasma high density lipoprotein level in diabetic and non-diabetic obese vagotomized rats. Vagotomy significantly decreased lee obesity index and perigonadal fat pads weight, decreased fasting insulinemia, decreased HOMA-IR and improved glucose tolerance in diabetic and non-diabetic obese vagotomized rats. These results were confirmed by regression of degenerative changes and restoration of the size of pancreatic islets of Langerhans. In non-diabetic obese vagotomized group, the results of 120 minutes of oral glucose tolerance test and HOMA-IR were close to normal expected values. However, the same parameters were still away from normal expected values although normal histopathological results of pancreases, in diabetic obese vagotomized group. In Conclusion, subdiaphragmatic vagotomy has a promising role in treating obesity. Nevertheless, its role in treating diabetes needs further long-term studies to be approved.

Corresponding author: Shimaa Mohammad Yousof; Department of Medical Physiology, Faculty of Medicine, Suez Canal University, Ismailia. E-mail: drshimaay@gmail.com, shimaam.yousof@med.suez.edu.eg 


\section{Introduction}

Diabetes mellitus (DM) is a chronic metabolic disease characterized by hyperglycemia as result of insulin resistance and a relative deficiency of insulin (1). More than $90 \%$ from all patients with DM have type 2 diabetes mellitus (T2DM) and the majority of them are obese (2). The prevalence of T2DM in Egypt was around $15.6 \%$ of all adults in 2015 (3). Currently, the most effective methods of T2DM treatment are insulin, oral antidiabetic drugs, lifestyle changes and surgical procedures such as Roux-en-Y gastric bypass (RYGB) (2). Unfortunately, current medical treatment has been relatively unsatisfied as more than half of the patients cannot achieve the therapeutic goal (4).

The main pathophysiological feature of obesity and T2DM is hyperlipidemia which results from dysregulation of lipid metabolism and diagnosed by elevated plasma triglyceride (TG) level, increased plasma low density lipoprotein (LDL) level and decreased plasma high density lipoprotein (HDL) level (5). The hepatic afferent vagal nerve fibers detect FFA concentration in portal veins (6). Khound $\boldsymbol{e t}$ al., (2017) reported that subdiaphragmatic vagotomy significantly decreased hepatic TG content and caused inhibition of hepatic de novo lipogenic genes leading to a decrease in hepatic lipid accumulation in 22 weeks of HFD induced obese rats

The vagus nerve is the main nerve of the PNS and has an important role on gastric emptying, lipid metabolism, and secretion of gastric and pancreatic digestive juices and anabolic hormones (5). Subdiaphragmatic vagotomy is the surgical technique in which all subdiaphragmatic vagal nerve fibers are removed (7). The possible postoperative complications of vagotomy are gastric retention, post-vagotomy osmotic diarrhea, and postvagotomy hypergastrinemia (8). Recently, truncal vagotomy was performed for the treatment of marginal ulcers after RYGB surgery for morbid obesity $(7,9,10)$. Vagal nerve blockade device (VBloc) was developed to cause intermittent intraabdominal vagal blockage using electrical currents and is the first new obesity treatment device to receive FDA approval in 14 years (11).

Insulin release depends on blood glucose level, beta cell mass and neural control which is mediated through glucose sensing in the hypothalamus and brainstem (12). Glucagon like peptide (GLP-1) excites preganglionic vagal motor neurons supplying the pancreas stimulating insulin secretion (5). The increased vagal activity in the pancreases of monosodium glutamate (MSG) induced obesity in rats was reported as the cause of increased total pancreatic beta cell area leading to hyperinsulinemia (13).

The role of subdiaphragmatic vagotomy in body weight reduction is controversial. Since, The previous study demonstrated that VBloc device caused significant weight loss in moderate obese individuals (11). The suggested mechanisms that could lead to weight loss after vagotomy are loss of appetite, decreased food intake, delayed gastric emptying, and changes in some gastrointestinal hormones (10).

On the contrary, another previous study reported that vagal innervation of the liver, pancreas and duodenum is not important for the beneficial effects of RYGB on food intake, glycemic control, and body weight (14).

Seeking for new treatment approaches to improve hyperlipidemia is mandatory in obese and type 2 
diabetic patients. So in this study, we used a model of diabetic obese rats to assess the effect of subdiaphragmatic vagotomy on lipid profile and insulin resistance in an attempt to develop a new effective treatment of obesity-associated T2DM to improves $\mathrm{T} 2 \mathrm{DM}$ and decreases the risk of complications.

\section{Materials and Methods}

Experimental animals: Fifty Male Albino rats weighing $200 \pm 50 \mathrm{~g}$ were purchased from the Ophthalmology Research Institute in Giza. Rats were housed in Physiology Department, Faculty of Medicine, and Suez Canal University at controlled room temperature. They were left to acclimatize for one week before the beginning of the study. The study protocol was approved from the Ethics Committee, Faculty of Medicine; Suez Canal University, Egypt.

Experimental design: Rats were randomly divided into 5 groups 10 per each. Group I (Control sham), Group II (Control vagotomized), Group III (Nondiabetic obese vagotomized), Group IV (Diabetic obese sham), and Group V (Diabetic obese vagotomized)

\section{Induction of experimental hyperlipidemia in rats:}

Rats in the group III, IV, V were given HFD (40\% fat, $42 \%$ carbohydrate, $18 \%$ protein) as a total percentage of calories for 5 weeks $(15,16)$. The rats were considered hyperlipidemic when there was significant increase in the plasma lipid profile when compared with the control group $(17,18)$. After 5 weeks, the Lee obesity index of rats in the HFD group was calculated by dividing the cube root of $\mathrm{BW}(\mathrm{g})$ by nasoanal length $(\mathrm{cm})$ and multiplying the result by 1000 , and rats with lee obesity index greater than 310 were considered obese $(17,19)$.

Induction of diabetes mellitus: At $6^{\text {th }}$ week, 12 hours fasting, the rats were given a single intraperitoneal injection of STZ (sigma-Aldrich, USA catalog No: 130, supplied in powder form) in a dose of $30 \mathrm{mg} / \mathrm{kg}$ to induce DM $(15,16)$. During the first 24 hours after the STZ intraperitoneal injection, rats were given $5 \%$ glucose solution. After 72 hours, rats with blood glucose levels more than or equal $200 \mathrm{mg} / \mathrm{dl}(11.1 \mathrm{mmol} / \mathrm{L})$ were considered diabetic rats and were assigned to the study $(15,20)$.

Subdiaphragmatic vagotomy procedure : After 12 hours of fasting, rats were anesthetized with intraperitoneal injection of a mixture of ketamine in a dose of $90 \mathrm{ml} / \mathrm{Kg}$ and xylazine in a dose of 9 $\mathrm{mg} / \mathrm{kg}$. Midline upper abdominal incision was made, and both anterior and posterior vagus nerves were separated from the esophagus and cut off $(19,21)$. After the operation, rats were received systemic analgesic (Ibuprofen $5 \mathrm{mg} / \mathrm{kg} \mathrm{SC}$ ) for 3 days in order to decrease postoperative pain (22) and subcutaneous $10 \mathrm{~cm}$ glucose $5 \%$ (Figure 1).

Sham operation: Rats were subjected to the same surgical procedures of vagotomy, but the vagus nerve wasn't cut off.

Oral glucose tolerance test (OGTT): Eight weeks after vagotomy, a blood sample was taken from the tail tip after 12 hours of fasting, to obtain fasting blood glucose level (time 0) using a glucometer (OK-1 meter, Biotech CO, Ltd). Then, all rats were given oral gavage of glucose in dose of 2 $\mathrm{g} / \mathrm{kg}$, and blood glucose levels at 15, 30, 60, 120 min after gavage were measured by glucometer from tail tip blood $(20,21)$. 
Insulin sensitivity: The homeostasis model of assessment of insulin resistance [(HOMA-IR) was measured according to the following formula:

HOMA-IR = fasting insulin $(\mathrm{mIU} / \mathrm{L}) \times$ fasting glucose $(\mathrm{mmol} / \mathrm{L}) / 22.5(13,20)$

\section{At the end of the study (8 weeks after vagotomy):}

Rats were euthanized with a combination of 100 $\mathrm{mg} / \mathrm{kg}$ of ketamine and $10 \mathrm{mg} / \mathrm{kg}$ of xylazine (16). Then, blood samples were taken by heart puncture for insulin measurement by ELISA (RayBio®, USA, Catalog No: ELR-Insulin) according to the manufacturer's instructions and lipid profile (CHOL, TG, HDL and LDL) by an automatic biochemistry analyzer. After sacrification, the retroperitoneal and perigonadal fat pads from all groups of rats were removed and weighed for assessment of obesity. The stomach food retention from all operated groups were assessed by the ratio between the stomach weight per body weight to confirm vagotomy $(19,21)$.

\section{Statistical analysis:}

Data were reported as mean $\pm \mathrm{SD}$ and were analyzed with SPSS statistical software version 20. One way ANOVA was used for comparing means of a variable in the five groups.

\section{Results}

Body weight, lee obesity index and perigonadal and retroperitoneal fat pads weight results:

Subdiaphragmatic vagotomy decreased BW, lee groups (Table 1). obesity index and perigonadal fat pads weight in diabetic and non-diabetic obese vagotomized rats.

The BW progression in HFD fed groups was significantly higher than control groups after 5 weeks of HFD ( $p$ value $<0.0001$ ). The BW of diabetic and non-diabetic obese vagotomized groups was significantly lower than of diabetic obese sham group at the end of experiment ( $p$ value $<0.0001$; Figure 2). There was significant decrease in BW of diabetic obese vagotomized group when compared with nondiabetic obese vagotomized groups ( $\mathrm{p}$ value $<$ 0.0001).

Lee obesity index, before vagotomy, was significantly higher in HFD fed groups; diabetic obese vagotomized group, diabetic obese sham group, and non-diabetic obese vagotomized group, when compared to control sham group ( $\mathrm{p}$ value $<$ 0.0001). The lee index of diabetic obese vagotomized group and non-diabetic obese vagotomized group was significantly decreased when compared with diabetic obese sham group $(\mathrm{p}$ value $<0.0001$, p value $<0.019$; respectively; Table 1). Vagotomy significantly decreased perigonadal fat pads weight in diabetic and non- diabetic obese vagotomized rats when compared with diabetic obese sham group ( $\mathrm{p}$ value $<0.0001$ and $\mathrm{p}$ value $<$ 0.02 respectively; Table $\mathbf{1})$.

Stomach weight (g) / BW (g) ratio to confirm subdiaphragmatic vagotomy

All vagotomized groups presented with higher stomach weight/ BW ratios than sham 
Table (1): The effect of vagotomy on stomach weight (g) / BW (g) ratio \& obesity parameters

\begin{tabular}{|l|c|c|c|c|c|c|}
\hline & Control sham & $\begin{array}{c}\text { Control } \\
\text { vagotomized }\end{array}$ & $\begin{array}{c}\text { Non-diabetic obese } \\
\text { vagotomized }\end{array}$ & $\begin{array}{c}\text { Diabetic obese } \\
\text { sham }\end{array}$ & $\begin{array}{c}\text { Diabetic obese } \\
\text { vagotomized }\end{array}$ \\
\hline $\begin{array}{l}\text { Stomach } \\
\text { weight }(\mathrm{g}) / \mathrm{BW} \quad(\mathrm{g})\end{array}$ & $0.75 \pm .05 \%$ & $0.96 \pm .05 \% *$ & $0.92 \pm .08 \% * \#$ & $.64 \pm .05 \%$ & $1 \pm .08 \% * \#$ \\
ratio & Pre & $263.2 \pm 6$ & $265.70 \pm 6$ & $313.1 \pm 3 *$ & $314.3 \pm 2.8 *$ & $314.1 \pm 3.35 *$ \\
\hline $\begin{array}{l}\text { Lee obesity } \\
\text { index }\end{array}$ & post & $273.3 \pm 3.9$ & $267.40 \pm 3$ & $293.4 \pm 13.17 \#$ & $307.5 \pm 15.2 *$ & $280.8 \pm 7.06 \# \$$ \\
\hline $\begin{array}{l}\text { Perigonadal fat } \\
\text { pads weight (g) }\end{array}$ & $1.44 \pm 0.1$ & $0.8 \pm 0.21 *$ & $3.64 \pm 0.33 \#$ & $3.93 \pm 0.095^{*}$ & $0.7 \pm 0.18 \# * \$$ \\
\hline Fasting insulinemia & $1.33 \pm 0.08$ & $0.52 \pm 0.11 *$ & $1.62 \pm 0.12 \#$ & $2.59 \pm 0.2 *$ & $1.86 \pm 0.17 \# \$$ \\
\hline
\end{tabular}

* Significant when compared to control sham group, \# Significant when compared to diabetic obese sham group, $\$$ Significant when compared to non-diabetic obese vagotomized group $(\mathrm{p} \leq 0.05$, one-way ANOVA followed by post Hoc tukey test).

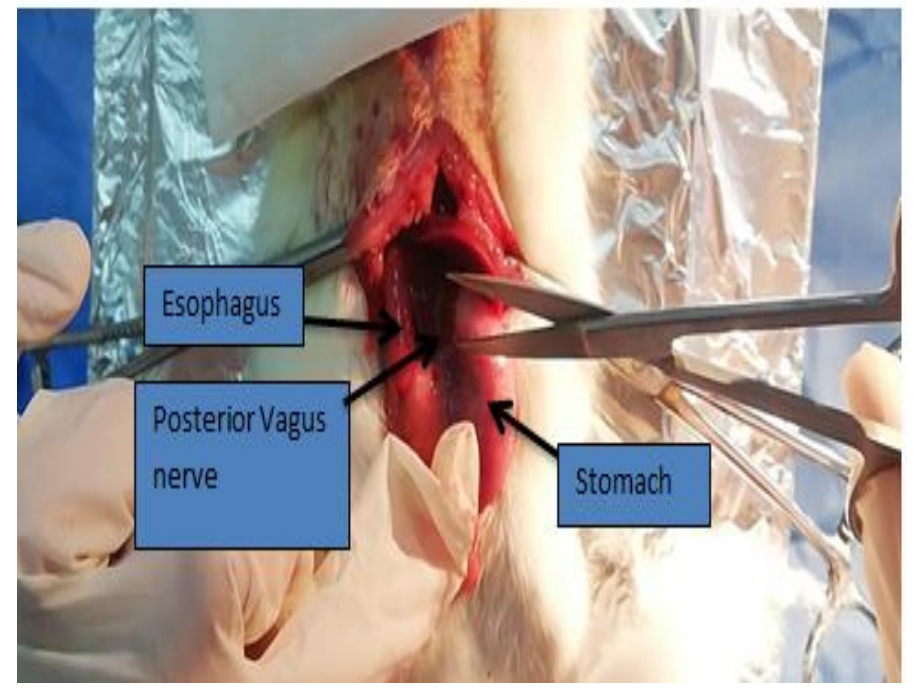

Figure (1)

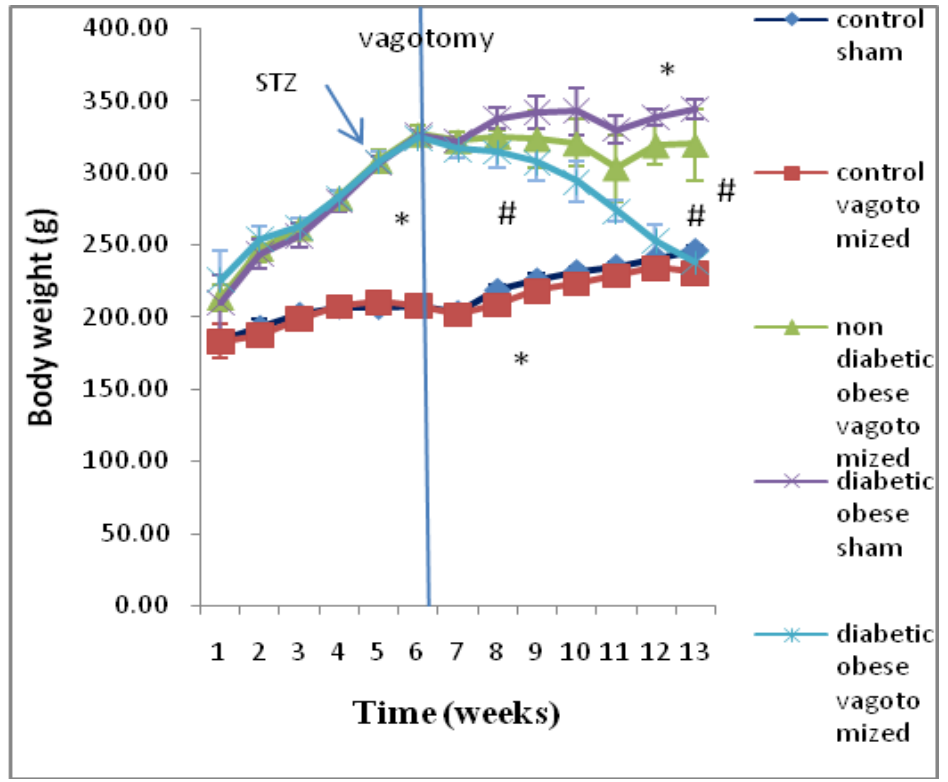

Figure (2): Body weight progression during 13 weeks of HFD feeding and the effect of subdiaphragmatic vagotomy on it. 


\section{Serum lipid profile}

Subdiaphragmatic vagotomy significantly decreased plasma CHOL, decreased plasma $\mathrm{TG}$, and increased plasma HDL in nondiabetic and diabetic obese vagotomized rats comparing to diabetic obese sham group ( $p$ value $<0.01$; Table 2). There was no significant difference between diabetic and non-diabetic obese vagotomized groups when compared with control sham group indicating that results of diabetic and non-diabetic obese vagotomized groups became close to normal results. Vagotomy did not affect plasma LDL level in diabetic obese vagotomized group although it significantly reduced LDL level in non-diabetic obese vagotomized group (p value $<0.01)$.
Oral glucose tolerance test, glycemia, fasting insulinemia and HOMA-IR

Before vagotomy, there was significant difference in glycemia between diabetic rat groups and non-diabetic rat groups ( $\mathrm{p}$ value $<0.00001)$.After vagotomy, There was significant decrease in fasting blood glucose in diabetic obese vagotomized when compared with diabetic obese sham group ( $p$ value $<0.04$; Figure 3).

During OGTT, vagotomy was found to significantly decrease blood glucose levels at 15, 60, and 120 minutes after glucose administration in diabetic obese vagotomized group when compared to diabetic obese sham group ( $p$ value $<0.023$, $p$ value $<0.0001$ and $p$ value $<0.0001$ respectively; Figure 4)

Table (2): Serum lipid profile before and after Subdiaphragmatic vagotomy

\begin{tabular}{|c|c|c|c|c|c|c|}
\hline & & $\begin{array}{l}\text { Control } \\
\text { sham }\end{array}$ & $\begin{array}{l}\text { Control } \\
\text { vagotomized }\end{array}$ & $\begin{array}{l}\text { Non-diabetic obese } \\
\text { vagotomized }\end{array}$ & $\begin{array}{l}\text { Diabetic obese } \\
\text { sham }\end{array}$ & $\begin{array}{l}\text { Diabetic obese } \\
\text { vagotomized }\end{array}$ \\
\hline \multirow{2}{*}{$\begin{array}{l}\text { Plasma } \\
\text { CHOL } \\
\text { (mg/dl) }\end{array}$} & Before & $24.7 \pm 0.82$ & $25.1 \pm 0.74$ & $65.4 \pm 3.17 *$ & $66.4 \pm 1.27 *$ & $66.9 \pm 0.99 *$ \\
\hline & After & $29.2 \pm 1.932$ & $25 \pm 1.054 *$ & $48.2 \pm 2.936 * \#$ & $79 \pm 1.054 *$ & $38.1 \pm 1.37 * \#$ \\
\hline \multirow{2}{*}{$\begin{array}{l}\text { Plasma TG } \\
(\mathrm{mg} / \mathrm{dl})\end{array}$} & Before & $15.8 \pm 1.14$ & $15.5 \pm 1.08$ & $129.3 \pm 1.34 *$ & $128.8 \pm 0.94 *$ & $127.9 \pm 0.88^{*}$ \\
\hline & After & $29.7 \pm 1.767$ & $16.7 \pm 2.584 *$ & $27.9 \pm 1.663 \#$ & $96.7 \pm 3.302 *$ & $28.7 \pm 1.252 \#$ \\
\hline \multirow{2}{*}{$\begin{array}{l}\text { Plasma HDL } \\
(\mathrm{mg} / \mathrm{dl})\end{array}$} & Before & $11.9 \pm 0.88$ & $11.8 \pm 0.92$ & $31.9 \pm 1.29 *$ & $32.1 \pm 1.2 *$ & $32.4 \pm 1.27 *$ \\
\hline & After & $20 \pm 1.054$ & $31.4 \pm 1.506^{*}$ & $29.7 \pm 1.059 *$ & $32.1 \pm 1.197 *$ & $42.6 \pm 1.174 * \#$ \\
\hline \multirow{2}{*}{$\begin{array}{l}\text { Plasma LDL } \\
(\mathrm{mg} / \mathrm{dl})\end{array}$} & Before & $1.2 \pm 0.79$ & $1.1 \pm 0.74$ & $22.3 \pm 1.25 *$ & $21 \pm 0.82 *$ & $22.1 \pm 0.88^{*}$ \\
\hline & After & $5.6 \pm 1.43$ & $3.8 \pm 1.398^{*}$ & $6.2 \pm 1.398 * \#$ & $11 \pm 1.054 * *$ & $10.8 \pm 1.476 * \$$ \\
\hline
\end{tabular}

CHOL: Cholesterol, TG: Triglycerides, HDL: High density lipoprotein, and LDL: Low density lipoprotein.* Significant when compared to control sham group, ** Significant when compared to control vagotomized group, \$ Significant when compared to non-diabetic obese vagotomized group, \# Significant when compared to diabetic obese sham group ( $\mathrm{p} \leq$ 0.05 , one-way ANOVA followed by post Hoc tukey test). 
There was no significant difference between non-diabetic obese vagotomized group and control vagotomized at 120 minutes. Vagotomy significantly decreased fasting plasma insulin in diabetic and non-diabetic obese vagotomized groups when compared to diabetic obese sham ( $p$ value $<0.0001$; Table 1). There was significant decrease in HOMAIR in diabetic and non-diabetic obese vagotomized groups when compared with diabetic obese sham group ( $\mathrm{p}$ value $<0.0001$, vagotomized group was still significantly higher than control sham group (p value $<0.0001)$.

\section{Histopathology of pancreses:}

Diabetic obese sham group revealed showed shrinkage of islets of Langerhans and fatty infiltration of exocrine portion. Restoration of the pancreatic islet structure was restored afer subdiaphragmatic vagotomy, (Figure 6,7,8).

Figure 5), but HOMA-IR in diabetic obese

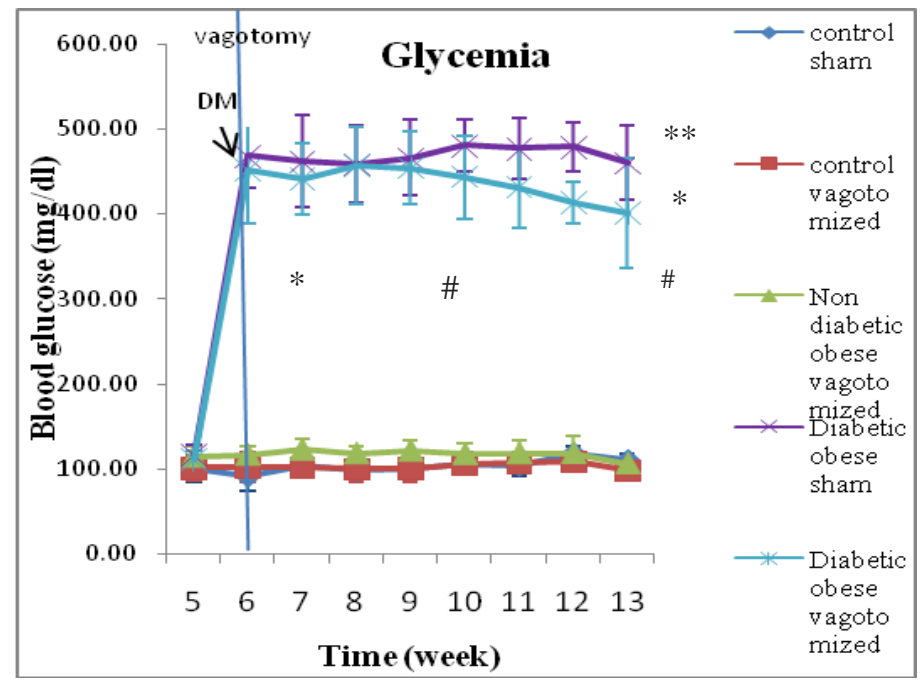

Figure (3): Blood glucose levels in rat groups after induction of DM.

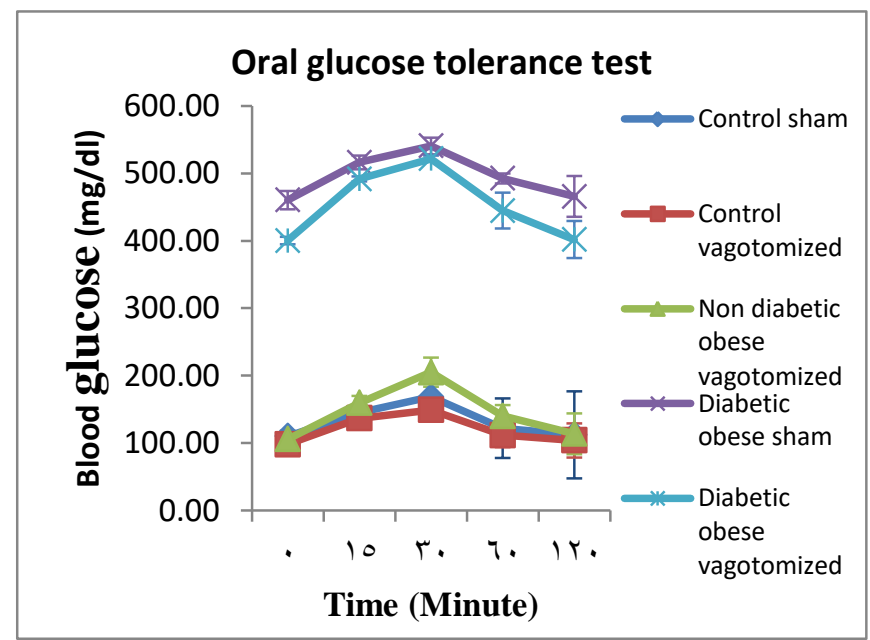

Figure (4): Changes in blood glucose levels during oral glucose tolerance test 8 weeks after subdiaphrgmatic vagotomy. 


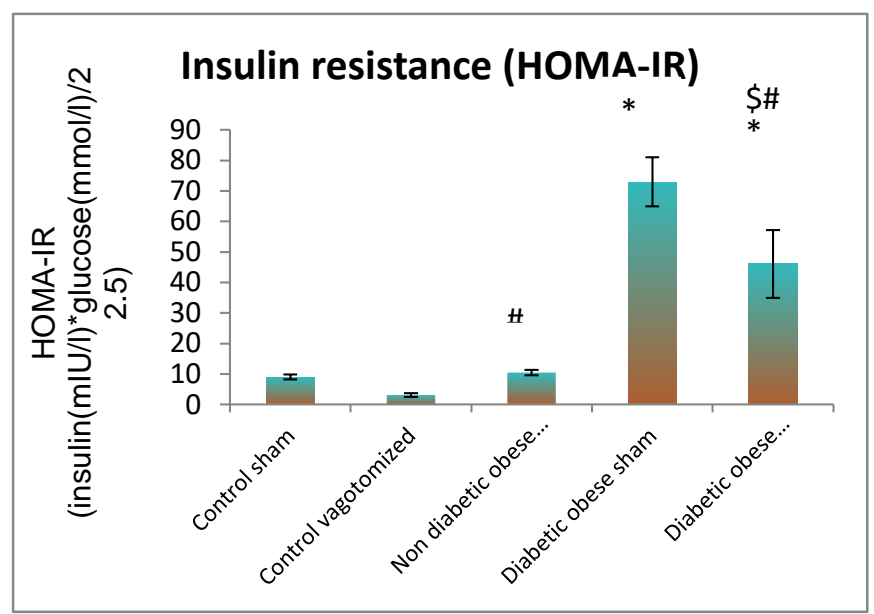

Figure (5): HOMA-IR (the homeostasis model of assessment of insulin Resistance)

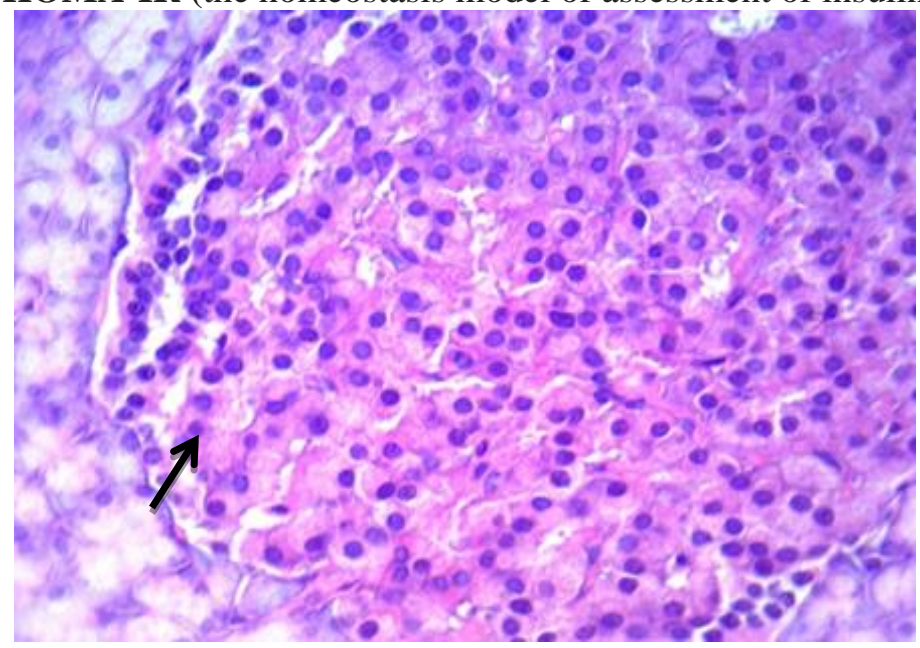

Figure (6): Photomicrographs of Sections in normal pancreatic tissue of control vagotomized rats showing large well defined islet of Langerhans (arrow) composed of regular rounded to cuboidal cells with eosinophilic cytoplasm and regular central rounded nuclei with trabecular or acinar arrangement. No degenerative changes. The pancreatic islet architecture was not changed between control sham and vagotomized groups (H\&Ex400).

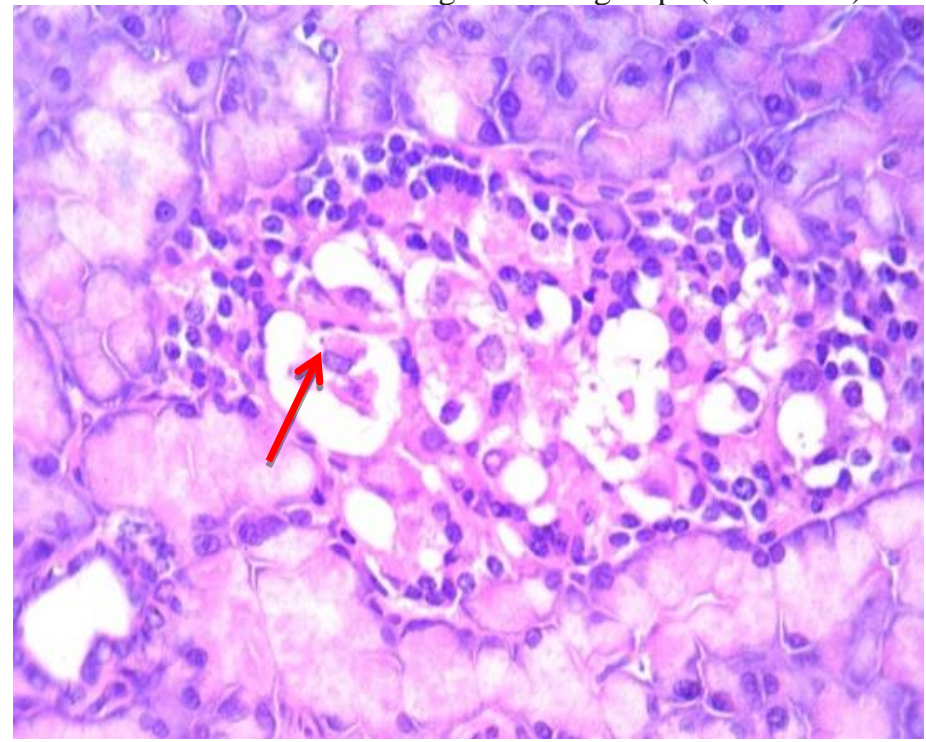

Figure(7): Photomicrographs of Sections in pancreatic tissue of diabetic obese sham group showing moderate shrinkage of islets with moderate to marked vacuolar degeneration (red) of cells and cytoplasmic fragmentation (H\&Ex400). 


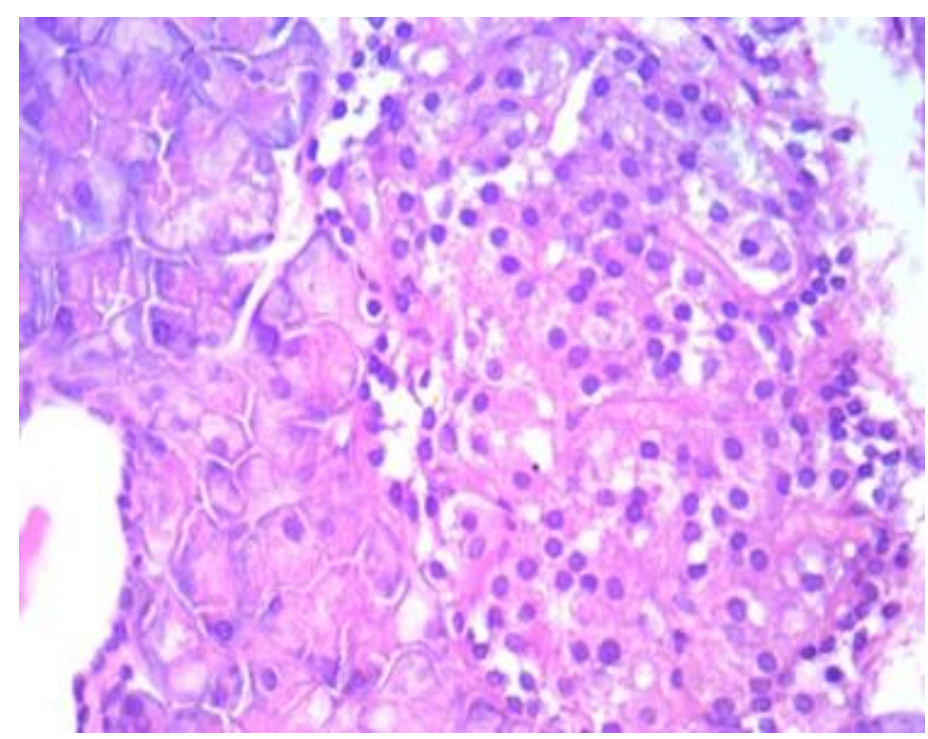

Figure (8): Photomicrographs of Sections in pancreatic tissue of diabetic obese vagotomized group showing marked improvement of degenerative changes, almost restored pancreatic islets size, regular cells with regular rounded nuclei, no fatty infiltration or degenerative changes, still noticed very focal minimal cytoplasmic vacuolation (H\&Ex400) .

\section{Discussion}

Type 2 diabetes mellitus is closely linked to obesity where there is insulin resistance and impaired lipid metabolism (23). HFD and low dose STZ induced diabetic obese rat model was applied in this study to promote obesity, insulin resistance and T2DM. This model was selected and applied to simulate the natural history and metabolic characteristics of T2DM in human (15).

Several studies have shown that ANS imbalance has an important role in pathophysiology of obesity. Increased vagal activity was found in HFD induced obese rat model to maintain obesity vicious cycle of hyperinsulinemia, insulin resistance, hyperlipidemia, and failure of beta cells leading to DM (24-26).

The present study demonstrated that subdiaphragmatic vagotomy improved plasma lipid profile, decreased perigonadal fat pads weight and decreased lee obesity index in diabetic and non-diabetic obese rats. Vagotomy normalized insulin resistance, improved glucose tolerance and restored normal size of pancreatic islets of Langerhans in non-diabetic obese rats. Previous studies showed similar results to the current one, Balbo et al., (2016) showed that vagotomy in cafeteria diet induced obesity in rats effectively decreased body weight and perigonadal fat stores, decreased insulinemia and improved insulin sensitivity. These effects were attributed to decreased cholinergic potentiation of insulin release. Morton et al., (2016) demonstrated that vagal nerve blockade caused significant weight loss in moderate obese humans. On the contrary of the previous results, Shin, 
Zheng and Berthoud, (2012) reported that hepatic branch vagotomy did not make significant difference in body weight and adiposity between RYGB with hepatic branch vagotomy and RYGB alone in obese rats. This controversy may be explained by the difference in methodology as the former study included vagotomized group with additional surgical procedure. In addition, the animals have been subjected to selective hepatic branch vagotomy in which hepatic branch of vagus nerve was removed theoretically. Meanwhile, in the present study, both anterior and posterior branches of subdiaphragmatic vagus nerve have been cut $(19,21)$.

Present study results demonstrated that diabetic obese vagotomized rats presented with more weight loss than nondiabetic obese rats. Interestingly, Li et al., (2014) reported concordant results on human subjects; they showed that that T2DM patients lost more weight than nondiabetic subjects during 16 weeks of behavioral weight loss program. Li et al., (2014) study explained that T2DM patients lost more calories in the urine than nondiabetic subjects contributing to more weight loss in diabetic patients. On the contrast to the current study results, Antoun et al., (2018) reported that T2DM patients have lost less weight than non-diabetic obese subjects. The differences in results noticed between Li et al., (2014) and Antoun et al., (2018) studies could be due to difference in initial body weight; suggesting that, starting with greater body weight in T2DM patients has been associated with more weight loss.

The present study demonstrated that subdiaphragmatic vagotomy normalized plasma TG level, decreased plasma $\mathrm{CHOL}$ level and increased plasma HDL level in diabetic and non-diabetic obese vagotomized rats after eight weeks. It also reported that non-diabetic obese vagotomized rats presented with reduced plasma LDL level, with no effect on plasma LDL level in diabetic obese rats. In agreement with this study, Lubaczeuski et al., (2015) demonstrated that subdiaphragmatic vagotomy interrupted the vagal afferent input to the CNS and vagal efferent input to the liver leading to decreased hepatic TG production and plasma TG level in monosodium glutamate induced obese vagotomized rats. Another study agreed with our results has reported that vagotomy reduced TG levels and VLDL production via elevation of plasma GLP-1 levels which mediates the brain liver vagal signals in decreasing VLDL production and insulin resistance induced by HFD in rats (5)

The improvement of plasma lipid profile was associated with improvement of 
fasting insulinemia, insulin resistance and subsequently glucose tolerance since, our study demonstrated that diabetic obese vagotomized rats significantly presented with lower fasting insulinemia, HOMA-IR and glycemia than diabetic obese sham operated rats. Qiu et al., (2014) reported that selective hepatic branch vagotomy significantly decreased fasting insulinemia in rats with $\mathrm{T} 2 \mathrm{DM}$ and these findings are concordant with the current study findings. Conversely, this previous study reported that hepatic branch vagotomy did not affect fasting glycaemia in rats with T2DM when compared with sham group and these findings are against our's. This controversy may be due to different T2DM model induction method. Qiu et al., (2014) induced T2DM in rats by single intraperitoneal injection of STZ $(60 \mathrm{mg} / \mathrm{kg})$ followed by nicotinamide $(120 \mathrm{mg} / \mathrm{kg})$.

The current study demonstrated that subdiaphragmatic vagotomy improved glucose tolerance in non-diabetic obese rats since; there was no significant difference in OGTT all times within 120 minutes of OGTT between non-diabetic obese vagotomized and control sham groups. These results were associated with normalization of fasting insulinemia, HOMA-IR and histopathological results of pancreases of non-diabetic obese vagotomized group. In agreement with current research, Lubaczeuski et al., (2015) reported that subdiaphragmatic vagotomy normalized glucose tolerance using intraperitoneal GTT due to normalization of insulinemia and HOMA-IR as result of restoration of normal morphofunctional aspects in pancreatic beta cells in MSG induced obese rats. Another previous study, in agreement with these observations, reported that subdiaphragmatic vagotomy improved glucose tolerance and enhanced hepatic insulin sensitivity via increased expression of insulin signaling molecules in the liver in HFD fed mice (21). In contrast to the current work results, Barella et $\boldsymbol{a l}$., (2015) documented that subdiaphragmatic vagotomy did not affect glucose tolerance in HFD fed rats. This controversy may be attributed to using intravenous route of administration of glucose during GTT, which allowed rapid diffusion of glucose without passing through GIT. This previous study applied vagotomy before HFD intake, in contrast to our methodology as we applied vagotomy after 5 weeks of HFD and continued HFD until the end of study.

Supdiaphragmatic vagotomy, in the present work, was more effective in ameliorating obesity than obesity with DM as there was significant difference between diabetic obese vagotomized group and nondiabetic obese vagotomized group in improving HOMA-IR, glycemia and OGTT 
at 8 weeks postvagotomy. On the contrary, Sathananthan et al., (2014) reported that vagal blockade in non-diabetic patients did not affect gastric emptying, glucose metabolism and insulin secretion. Shikora et al., (2015) reported that intermittent vagal blockade which was applied on patients with T2DM caused weight loss, decreased blood pressure and $\mathrm{HbA} 1 \mathrm{c}$ reduction.

Points that could give a strength to the present study is the study design which included more than control one group as well as the confirmation of improvement in insulin resistance by regain of normal histopathological architecture of pancreases. A limitation of the current study is short time for induction of obesity. Another limitation of this study is short time of follow up after vagotomy.

In summary, subdiaphragmatic vagotomy is effective in improving plasma lipid profile, decreasing HFD induced hyperinsulinemia and decreasing body weight in both diabetic and non-diabetic obese rats. Nevertheless, prolongation of periods of follow up after subdiaphragmatic vagotomy might be needed for assessment of the long term effects of subdiaphragmatic vagotomy on insulin resistance and glucose tolerance in diabetic obese rats.

\section{Recommendations:}

Further studies are required to elucidate the underlying mechanisms. VBloc device should be considered in Egypt for upgrading research in vagal nerve inhibition in treating metabolic disorders.

\section{Conflict of interest:}

The authors declare no conflicts of interest.

\section{References}

1. Ogurtsova K, da Rocha Fernandes JD, Huang Y, Linnenkamp U, Guariguata L, Cho NH, et al. IDF Diabetes Atlas: Global estimates for the prevalence of diabetes for 2015 and 2040. Diabetes Res Clin Pract. Elsevier; 2017;128:40-50.

2. Gao Z, Wang B, Gong X, Yao C, Ren D, Shao L, et al. Effect of gastric bypass combined with ileal transportation on type 2 diabetes mellitus. Exp Ther Med. 2018;15(5):4571-7.

3. Hegazi R, El-Gamal M, Abdel-Hady N, Hamdy O. Epidemiology of and Risk Factors for Type 2 Diabetes in Egypt. Ann Glob Heal. Elsevier; 2015;81(6):814-20.

4. Lee W-J, Chong K, Aung L, Chen SC, Ser K-H, Lee Y-C. Metabolic surgery for diabetes treatment: sleeve gastrectomy or gastric bypass? World $\mathrm{J}$ Surg. Springer; 2017;41(1):216-23.

5. Khound R, Taher J, Baker C, Adeli K, Su Q. GLP-1 elicits an intrinsic gut- 
liver metabolic signal to ameliorate diet-induced VLDL overproduction and insulin resistance. Arterioscler Thromb Vasc Biol. 2017;37(12):2252-9.

6. Mizuno K, Ueno $\mathbf{Y}$. Autonomic nervous system and the liver. Hepatol Res. Wiley Online Library; 2017;47(2):160-5.

7. Dezfuli G, Gillis RA, Tatge JE, Duncan KR, Dretchen KL, Jackson PG, et al. Subdiaphragmatic Vagotomy With Pyloroplasty Ameliorates the Obesity Caused by Genetic Deletion of the Melanocortin 4 Receptor in the Mouse. Front Neurosci [Internet]. 2018;12(March):104. Available from: http://journal.frontiersin.org/article/10.3 389/fnins.2018.00104/full

8. Seeras K, Prakash S. Truncal Vagotomy. In: StatPearls [Internet]. StatPearls Publishing; 2018.

9. Starup-Linde J, Gejl M, Borghammer P, Knop FK, Gregersen S, Rungby J, et al. Vagotomy and subsequent development of diabetes - A nested case-control study. Metabolism [Internet]. Elsevier Inc.; 2016;65(7):954-60. Available from: http://dx.doi.org/10.1016/j.metabol.201 6.04 .002

10. Liu T, Zhong MW, Liu Y, Huang X, Cheng YG, Wang KX, et al. Effects of sleeve gastrectomy plus trunk vagotomy compared with sleeve gastrectomy on glucose metabolism in diabetic rats. World J Gastroenterol. 2017;23(18):3269-78.

11. Morton JM, Shah SN, Wolfe BM, Apovian CM, Miller CJ, Tweden KS, et al. Effect of Vagal Nerve Blockade on Moderate Obesity with an ObesityRelated Comorbid Condition: the ReCharge Study. Obes Surg [Internet]. Obesity Surgery; 2016;26(5):983-9. Available from: http://dx.doi.org/10.1007/s11695-0162143-y

12. Carnagarin R, Matthews VB, Herat LY, Ho JK, Schlaich MP. Autonomic Regulation of Glucose Homeostasis : a Specific Role for Sympathetic Nervous System Activation. Current Diabetes Reports; 2018;

13. Lubaczeuski C, Balbo SL, Ribeiro RA, Vettorazzi JF. Vagotomy ameliorates islet morphofunction and body metabolic homeostasis in MSGobese rats. 2015;48:447-57.

14. Shin AC, Zheng H, Berthoud H-R. Vagal innervation of hepatic portal vein and liver is not necessary for Roux-enY gastric bypass surgery-induced hypophagia, weight loss, and hypermetabolism. Ann Surg. NIH Public Access; 2012;255(2):294.

15. Han H, Hu C, Wang L, Zhang G, Liu S, Li F, et al. Duodenal-jejunal bypass surgery suppresses hepatic de novo 
lipogenesis and alleviates liver fat accumulation in a diabetic rat model. Obes Surg. Springer; 2014;24(12):2152-60.

16. Ranjbar Kohan N, Nazifi S, Tabandeh MR, Ansari Lari M. Effect of L-Carnitine Supplementation on Apelin and Apelin Receptor (Apj) Expression in Cardiac Muscle of Obese Diabetic Rats. Cell J [Internet]. 2018;20(3):427-34. Available from: http://www.ncbi.nlm.nih.gov/pubmed/2 9845798\%0Ahttp://www.pubmedcentra 1.nih.gov/articlerender.fcgi?artid=PMC6 005003

17. Hariri N, Thibault L. High-fat dietinduced obesity in animal models. Nutr Res Rev. Cambridge University Press; 2010;23(2):270-99.

18. Defo PBD, Wankeu-Nya M, Esther, Ngadjui, Fozin GRB, Xavier F, et al. Palm Oil Diet-Induced Obesity Impairs Male Rat Reproductive Performance. Ann Reprod Med Treat. 2017;2(2):1012.

19. Balbo SL, Ribeiro RA, Mendes MC, Lubaczeuski C, Maller ACPA, Carneiro EM, et al. Vagotomy diminishes obesity in cafeteria rats by decreasing cholinergic potentiation of insulin release. J Physiol Biochem. Springer; 2016;72(4):625-33.

20. Ghelani H, Razmovski-Naumovski V, Nammi S. Chronic treatment of (R)- $\alpha-$ lipoic acid reduces blood glucose and lipid levels in high-fat diet and lowdose streptozotocin-induced metabolic syndrome and type 2 diabetes in Sprague-Dawley rats. Pharmacol Res Perspect. 2017;5(3):1-12.

21. Khound R. Effects of Vagotomy and Fenugreek on Hyperlipidemia and Insulin Resistance. 2017;

22. Waite ME, Tomkovich A, Quinn TL, Schumann AP, Dewberry L, Totsch SK, et al. Efficacy of common analgesics for postsurgical pain in rats. $\mathrm{J} A \mathrm{~m}$ Assoc Lab Anim Sci. American Association for Laboratory Animal Science; 2015;54(4):420-5.

23. Grayson BE, Seeley RJ, Sandoval DA. Wired on sugar: the role of the CNS in the regulation of glucose homeostasis. Nat Rev Neurosci. Nature Publishing Group; 2013;14(1):24.

24. Skovsø S. Modeling type 2 diabetes in rats using high fat diet and streptozotocin. J Diabetes Investig. Wiley Online Library; 2014;5(4):34958.

25. Barella LF, Miranda RA, Franco CCS, Alves VS, Malta A, Ribeiro TAS, et al. Vagus nerve contributes to metabolic syndrome in high-fat diet-fed young and adult rats. Exp Physiol. 2015;100(1):57-68.

26. Masi EB, Valdés-Ferrer SI, Steinberg BE. The vagus neurometabolic 
interface and clinical disease. Int J Obes

[Internet]. Springer US; 2018;42(6):1101-11. Available from: http://dx.doi.org/10.1038/s41366-0180086-1

27. Li Z, Tseng CH, Li Q, Deng ML, Wang M, Heber D. Clinical efficacy of a medically supervised outpatient highprotein, low-calorie diet program is equivalent in prediabetic, diabetic and normoglycemic obese patients. Nutr Diabetes. Nature Publishing Group; 2014;4(2):e105.

28. Antoun G, Nikpay M, McPherson R, Harper M-E, Dent R. Is Type 2 Diabetes in Adults Associated With Impaired Capacity for Weight Loss? Can J diabetes. Elsevier; 2018;42(3):313-6.

29. Qiu NC, Zhang Q, Song X, Liu ME, Li XK, Shan CX, et al. Impact of the hepatic branch of the vagus and Rouxen-Y gastric bypass on the hypoglycemic effect and glucagon-like peptide- 1 in rats with type 2 diabetes mellitus. J Surg Res [Internet]. Elsevier Inc; 2014;191(1):123-9. Available from:

http://dx.doi.org/10.1016/j.jss.2014.03.0 62

30. Sathananthan M, Ikramuddin S, Swain JM, Shah M, Piccinini F, Dalla Man C, et al. The effect of vagal nerve blockade using electrical impulses on glucose metabolism in nondiabetic subjects. Diabetes, Metab Syndr Obes targets Ther. Dove Press; 2014;7:305.

31. Shikora SA, Wolfe BM, Apovian CM, Anvari M, Sarwer DB, Gibbons RD, et al. Sustained Weight Loss with Vagal Nerve Blockade but Not with Sham: 18-Month Results of the ReCharge Trial. J Obes. Hindawi Publishing Corporation; 2015;2015. 